\title{
Relation between Tolman length and isothermal compressibility for simple liquids*
}

\author{
Wang Xiao-Song(王小松) $)^{\mathrm{a} b)}$ and Zhu Ru-Zeng(朱如曾 $)^{\mathrm{b}) \dagger}$ \\ a) Institute of Mechanics and Power Engineering, Henan Polytechnic University, Jiaozuo 454003, China \\ ${ }^{b)}$ State Key Laboratory of Nonlinear Mechanics, Institute of Mechanics, Chinese Academy of Sciences, Beijing 100190, China
}

(Received 2 July 2012; revised manuscript received 8 August 2012)

The Tolman length $\delta_{0}$ of a liquid with a plane surface has attracted increasing theoretical attention in recent years, but the expression of Tolman length in terms of observable quantities is still not very clear. In 2001, Bartell gave a simple expression of Tolman length $\delta_{0}$ in terms of isothermal compressibility. However, this expression predicts that Tolman length is always negative, which is contrary to the results of molecular dynamics simulations (MDS) for simple liquids. In this paper, this contradiction is analyzed and the reason for the discrepancy in the sign is found. In addition, we introduce a new expression of Tolman length in terms of isothermal compressibility for simple fluids not near the critical points under some weak restrictions. The Tolman length of simple liquids calculated by using this formula is consistent with that obtained using MDS regarding the sign.

Keywords: Tolman length, isothermal compressibility, surface tension, thermodynamics

PACS: 68.03.Cd, 68.35.Md, 05.70.Np

\section{Introduction}

In 1949, Tolman obtained the following relation between the surface tension $\sigma$ and the radius of the surface of tension $R_{\mathrm{S}}$ for spherical droplets: $:^{[1]}$

$$
\sigma=\sigma_{0}\left(1-\frac{2 \delta_{0}}{R_{\mathrm{s}}}+\cdots\right)
$$

where $\sigma_{0}$ is the surface tension of the planar liquid surface, and $\delta_{0}$ is called the Tolman length for a planar surface, or Tolman length for short.

In theory,

$$
\delta_{0}=\lim _{R_{\mathrm{S}} \rightarrow \infty} \delta\left(R_{\mathrm{S}}\right)
$$

where $\delta\left(R_{\mathrm{S}}\right)$ is defined as

$$
\delta\left(R_{\mathrm{S}}\right)=R_{\mathrm{e}}-R_{\mathrm{S}},
$$

where $R_{\mathrm{e}}$ is the radius of the equimolar surface. ${ }^{[1]}$ Equation (1) shows that the surface tension deviates from its planar value when the droplet radius is on the order of the Tolman length, so that the Tolman length measures the extent to which the surface tension of a small liquid drop deviates from its planar value. Since any small radius depending on the surface tension influences the nucleation rate exponentially, experimental interest is increased due to its nucleation phenomena. ${ }^{[2]}$ The Tolman's length $\delta_{0}$ of a liquid with a plane surface has also caught increasing theoretical attention in recent years, while the expression of Tolman length in terms of the observable quantities is still not very clear. ${ }^{[3]}$ In 2001, Bartell's thermodynamic approximate treatment proposed a simple relation between the Tolman length and the isothermal compressibility at
DOI: $10.1088 / 1674-1056 / 22 / 3 / 036801$

a two-phase coexistence point in the form of

$$
\delta_{0} \approx-\kappa_{1} \sigma_{0}
$$

where $\kappa_{\mathrm{l}}$ is the isothermal compressibility of the bulk liquid phase. ${ }^{[4]}$ In 2006, Blokhuis and Kuipers ${ }^{[3]}$ gave an exact thermodynamic expression

$$
\delta_{0}=-\frac{\sigma_{0}}{\left(\Delta \rho_{0}\right) 2}\left[\rho_{1,0}^{2} \kappa_{1}-\rho_{\mathrm{v}, 0}^{2} \kappa_{\mathrm{v}}\right]-\frac{\mu_{2} \Delta \rho_{0}}{2 \sigma_{0}}
$$

based on an earlier thermodynamic expression ${ }^{[5-7]}$

$$
\delta_{0}=-\frac{\Delta \rho_{1}}{2 \Delta \rho_{0}}-\frac{\mu_{2} \Delta \rho_{0}}{2 \sigma_{0}}
$$

where $\kappa_{\mathrm{V}}$ is the isothermal compressibility of the bulk vapor phase at the two-phase coexistence point; $\rho_{\mathrm{l}, 0}$ and $\rho_{\mathrm{v}, 0}$ are the densities of the bulk liquid phase and the bulk vapor phase at the two-phase coexistence, respectively; $\Delta \rho_{0}$ and $\Delta \rho_{1}$ are the difference between the densities of bulk liquid phase and bulk vapor phase at the two-phase coexistence point and its first derivative with respect to the curvature of the equimolar surface at zero curvature, respectively; $\mu_{2}$ is half of the second derivative of the chemical potential with respect to curvature of the equimolar surface at zero curvature. In 2010, Zhu and Wang ${ }^{[8]}$ derived two general relationships between Tolman length and other thermodynamic quantities for singlecomponent liquid-vapor systems by replacing the equimolar surface with ordinary dividing surface. Equations (5) and (6) turn out to be two special cases of their results.

Since it is difficult to obtain the second derivatives of the chemical potential in Eq. (5) and to use Eq. (5), it is of

* Project supported by the National Natural Science Foundation of China (Grant No. 11072242).

${ }^{\dagger}$ Corresponding author. E-mail: zhurz@lnm.imech.ac.cn

다이 2013 Chinese Physical Society and IOP Publishing Ltd

http://iopscience.iop.org/cpb http://cpb.iphy.ac.cn 
great significance to find approximate formulas of the Tolman length and their application conditions. In this context, Blokhuis and Kuipers ${ }^{[3]}$ pointed out that Bartell's relation (4) could be obtained from Eq. (5) based on the following two assumptions:

$$
\begin{aligned}
& \mu_{2} \approx 0, \\
& p_{\mathrm{v}} \approx 0 .
\end{aligned}
$$

However, the Tolman length obtained with the help of Bartell's relation (4) is always negative, which contradicts with the values originating from MD simulations for simple liquid in the sign. MD simulations have been widely accepted as a standard test method for the Tolman length. ${ }^{[3,9-13]}$ For example, Blokhuis and Kuipers ${ }^{[3]}$ pointed out that the discrepancy in sign and its dependence on the interaction potential is not yet understood, and further MD simulations may help us to resolve these issues. Therefore, Bartell's relation (4) is incorrect for simple liquids at least.

In this paper, we analyze the cause of the discussed problem related to Bartell's formula (4) and propose a new approximate relation between the Tolman length and the isothermal compressibility for simple fluids not near the critical points, under some other weak restrictions. This relation can always result in a Tolman length which is consistent with all the results by means of MD simulations about the sign.

\section{Analysis of Bartell's formula}

On the one hand, we have analyzed the composition of Bartell's formula (4) above. Blokhuis and Kuipers pointed out that Eq. (5) is equivalent to Bartell's formula (4) under the assumptions (7) and (8). However, assumption (7) is not always correct for all systems. This is the reason for that the Tolman length obtained using Bartell's relation (4) is different from that derived by means of MD simulations.

On the other hand, we check Bartell's deriving procedure of Eq. (4). He adopted the following fundamental assumption:

$$
\Delta \mu=\sigma_{0} v_{1,0} / R_{\mathrm{e}}+\left(p_{\mathrm{v}}-p_{\mathrm{v}, 0}\right) v_{1,0},
$$

where $\Delta \mu$ is the difference between the liquid drop (at unstable equilibrium with the vapor at pressure $p_{\mathrm{v}}$ ) and the bulk liquid (at the bulk equilibrium vapor pressure $p_{\mathrm{v}, 0}$ ) in the chemical potential at the same temperature, and $v_{1,0}$ is the molar volume of the bulk liquid at the pressure $p_{\mathrm{v}, 0}$.

In order to reveal the possible essence of assumption (9), we note that it can be derived from the following two subassumptions. i) The liquid phase is incompressible, that is,

$$
v_{1}=v_{1,0},
$$

where $v_{1}$ is the molar volume of the internal phase of the liquid drop. ii)

$$
p_{1}-p_{\mathrm{v}}=2 \sigma_{0} / R_{\mathrm{e}}
$$

where $p_{1}$ is the pressure of the internal phase of the liquid drop.

Bartell computed the quantity $\Delta \mu$ using two different methods. One was based on the assumption (9), and the other was based on the relation

$$
\Delta \mu=\int_{p_{\mathrm{v}, 0}}^{p_{1}} v_{\mathrm{l}} \mathrm{d} p
$$

together with the state equation of linearly compressible fluids. Finally, he derived Eq. (4) by assuming that $\Delta \mu$ obtained from these two methods are equal.

In fact, $\Delta \mu$ obtained by these two methods are not equal, which can be proved as follows.

Firstly, we use sub-assumptions (10) and (11) to replace assumption (9) in the first method to check whether $\Delta \mu$ obtained through these two methods are equal.

Noting that Eq. (11) could also be described as ${ }^{[14]}$

$$
p_{1}-p_{\mathrm{v}}=2 \sigma_{\mathrm{e}} / R_{\mathrm{e}}+\left[\frac{\partial \sigma}{\partial R}\right]_{R=R_{\mathrm{e}}}=2 \sigma_{\mathrm{e}} / R_{\mathrm{e}}+\frac{\partial \sigma_{\mathrm{e}}}{\partial R_{\mathrm{e}}},
$$

where the square-bracketed term gives the derivative of $\sigma(R)$ with respect to a mathematical displacement of the dividing surface, we obtain

$$
\sigma_{\mathrm{e}}+\frac{R_{\mathrm{e}}}{2} \frac{\partial \sigma_{\mathrm{e}}}{\partial R_{\mathrm{e}}}=\sigma_{0}
$$

by combining Eq. (11) and Eq. (13) and

$$
\sigma_{\mathrm{e}}\left(R_{\mathrm{e}}\right)=\sigma_{0}+\frac{k_{\mathrm{s}}}{R_{\mathrm{e}}^{2}},
$$

where $k_{\mathrm{S}}$ is an integration constant ${ }^{[14]}$ obtained by integrating Eq. (14) at a constant temperature. Equation (15) implies that

$$
\delta_{0}=0
$$

which is contrary to Eq. (4). This contradiction demonstrates that $\Delta \mu$ obtained by these two methods are not equal.

Secondly, we analyze the assumption (9) as a whole. Assumption (9) is so unique and complex for us to understand it and to judge whether a given system satisfies it, unless it is divided into two sub-assumptions (10) and (11). Though some researchers believed that the validity of assumption (9) has been justified by some density functional theory (DFT) calculations for simple fluids, ${ }^{[15,16]}$ we would like to point out that because DFT contains some approximations, the conclusive judgment should be made by MD simulations. Since MD simulations are inconsistent with Bartell's solution (Eq. (4)) for simple liquids at least, they are also incompatible with assumption (9).

Therefore, the assumption that the values of $\Delta \mu$ obtained using the two methods are equal is incorrect. This explains the discrepancy in the sign of the Tolman length derived by Bartell's equation (4) and that given by MD simulations for simple liquids. 


\section{A new approximate relation between the Tol- man length and isothermal compressibility}

Since the known approximate relation between the Tolman length and isothermal compressibility (Eq. (4)) is invalid, it is meaningful to seek a new approximate relation between the Tolman length and isothermal compressibility, which is consistent with the results originating from MD simulations about the sign. In this section, we apply this idea to simple liquids not near the critical point under some other weak restrictions.

On the one hand, based on the Kirkwood-Buff molecular theory of the surface tension, assuming $\rho_{\mathrm{v} 0}=0$ and $g\left(z_{1}, z_{2}, r_{12}\right)=g_{10}\left(r_{12}\right)$, Jain and Nanda ${ }^{[17]}$ proved that

$$
\delta_{0}(w) \sigma_{0}(w)=\frac{\pi \rho_{1,0}^{2}}{30} \int_{0}^{\infty} r^{5} u^{\prime}(r) g_{1}(r) \mathrm{d} r,
$$

for the case of a linear or a cubic density profile of transition layer, where $u(r)$ is the pair potential, $g_{10}(r)$ stands for the two-body distribution function in the bulk liquid, and $w$ is the width of the transition zone.

Since the width of the transition zone $w$ is greater than several core diameters and not close to the critical point, $\rho_{\mathrm{v}, 0}=0$, the surface tension is approximately equal to

$$
\sigma_{0}(w) \approx \frac{A \rho_{1,0}^{2}}{w} \int_{0}^{\infty} r^{5} u^{\prime}(r) g_{1}(r) \mathrm{d} r
$$

where $A$ is a positive dimensionless parameter depending on the profile shape. ${ }^{[18,19]}$ It is worth noting that to understand the sufficiency of the condition that the width $w$ is larger than several core diameters, one can refer to Ref. [19], the authors of which stated that it is "good approximation even when $w$ equals the core diameter".

Combination of Eq. (17) and Eq. (18) gives

$$
\delta_{0}(w)=\frac{\pi}{30 A} w .
$$

We can see from Eq. (19) that $\delta_{0}(w)$ is proportional to $w$.

On the other hand, based on the density functional theory, Mon and Stroud gave the approximate relation between the width of the liquid-vapor transition zone and the isothermal compressibility of simple liquids ${ }^{[20]}$

$$
w=\frac{12 \rho_{1,0}^{2} \kappa_{\mathrm{l}} \sigma_{0}}{\left(\rho_{\mathrm{l}, 0}-\rho_{\mathrm{v}, 0}\right)^{2}} .
$$

Neglecting $\rho_{\mathrm{v}, 0}$ in Eq. (20) and substituting Eq. (20) into Eq. (19), we can obtain

$$
\delta_{0}(w)=\frac{2 \pi \kappa_{1} \sigma_{0}}{5 A}
$$

Obviously, Eq. (21) always gives a positive Tolman length, which is consistent with that given by molecular dynamic simulations for simple liquids about the sign. The positive parameter $A$ depends on the density profile of the transition zone. For the exponential density variation, Lekner and Henderson obtained $A=\pi / 30$ under the first-order approximation. ${ }^{[18]}$ We calculated $A$ based on Eq. (21) using the MD simulation results of surface tensions, the Tolman lengths for the Lennard-Jones potential, and the experimental data for the isothermal compressibility of Argon. ${ }^{[21]}$ The values of $A$ are listed in Table 1. Different values of the constant $A$ in Table 1 originate from different surface tensions and Tolman lengths obtained by different molecular dynamic simulations.

Table 1. Value of $A$ for Argon calculated by means of Eq. (21). Here, $T^{*}=k_{\mathrm{B}} T / \varepsilon$ is the reduced temperature, where $\varepsilon=119.8 K k_{\mathrm{B}}$ is the depth of the Lennard-Jones potential for Argon, and $k_{\mathrm{B}}$ is the Boltzmann constant; $\sigma_{0}^{*}=\varepsilon / d^{2}$ is the reduced surface tension, where $d=0.3405 \mathrm{~nm}$ is the length parameter of the Lennard-Jones potential for Argon; $\delta_{0}^{*}=\delta_{0} / d$ is the reduced Tolman length; $\kappa_{1}^{*}=\kappa_{1} \varepsilon / d^{3}$ is the reduced isothermal compressibility for liquid Argon.

\begin{tabular}{cccccc}
\hline & $T^{*}$ & $\kappa_{1}^{*}$ & $\sigma_{0}^{*}$ & $\delta_{0}^{*}$ & $A$ \\
\hline Ref. [13] & 0.80 & 0.1465 & 0.876 & 0.055 & 2.93 \\
Ref. [12] & 0.80 & 0.1465 & 0.391 & 0.169 & 0.42593 \\
Ref. [9] & 0.80 & 0.1465 & 0.388 & 0.14 & 0.51021 \\
\hline
\end{tabular}

\section{Conclusion}

The relation between the Tolman length and isothermal compressibility given by Bartell predicts that the Tolman lengths are always negative, which is contrary to the results of MD simulations for simple liquids in the sign. In this paper, we have analyzed the origin of this contradiction. Additionally, we have obtained a new approximate relation between the Tolman length and isothermal compressibility for simple fluids not near the critical points and under some other weak restrictions. Tolman lengths of simple liquids calculated by this formula are consistent with the results obtained by MD simulations at least in the sign.

\section{References}

[1] Tolman R C 1949 J. Chem. Phys. 17333

[2] Abraham F F 1974 Homogeneous Nucleation Theory (New York: Academic Press)

[3] Blokhuis E M and Kuipers J 2006 J. Chem. Phys. 124074701

[4] Bartell L S 2001 J. Phys. Chem. B 10511615

[5] Fisher M P A and Wortis M 1984 Phys. Rev. B 296252

[6] Blokhuis E M and Bedeaux D 1993 Mol. Phys. 80705

[7] Groenewold J and Bedeaux D 1995 Physica A 214356

[8] Zhu R Z and Wang X S 2010 Chin. Phys. B 19076801

[9] Haye M J and Bruin C 1994 J. Chem. Phys. 100556

[10] Bardouni H El, Mareschal M, Lovett R and Baus M 2000 J. Chem. Phys. 1139804

[11] Nijmeijer M J P, Bruin C, van Woerkom A B, Bakker A F and van Leeuwen J M J 1992 J. Chem. Phys. 96565

[12] van Giessen A E and Blokhuis E M 2002 J. Chem. Phys. 116302

[13] Lei Y A, Bykov T, Yooo S and Zeng X C 2005 J. Am. Chem. Soc. 127 15346

[14] McGraw R and Laaksonen A 1997 J. Chem. Phys. 1065284

[15] Laaksonen A and McGraw R 1996 Europhys. Lett. 35367

[16] Talanquer V and Oxtoby D W 1995 J. Phys. Chem. 992865

[17] Uang Y H 1980 Phys. Rev. A 22758

[18] Berry M V, Durran R F and Evens R 1972 J. Phys. A 5166

[19] Lekner J and Henderson J R 1977 Mol. Phys. 34333

[20] Mon K K and Stroud D 1980 Phys. Rev. Lett. 45817

[21] Jain S C and Nanda V S 1971 J. Phys. C 43045 